\title{
ON A SUBGROUP OF THE GROUP OF MULTIPLICATIVE ARITHMETIC FUNCTIONS
}

T. B. CARROLL and A. A. GIOIA

(Received 9 July 1974)

\section{Introduction}

An arithmetic function $f$ is said to be multiplicative if $f(1)=1$ and $f(m n)$ $=f(m) f(n)$ whenever $(m, n)=1$, where $(m, n)$ denotes as usual the greatest common divisor of $m$ and $n$. Furthermore an arithmetic function is said to be linear (or completely multiplicative) if $f(1)=1$ and $f(m n)=f(m) f(n)$ for all positive integers $m$ and $n$. The Dirichlet convolution of two arithmetic functions $f$ and $g$ is defined by

$$
f \circ g(n)=\sum_{a b=n} f(a) g(b)
$$

for all $n \in Z^{+}$. Recall that the set of all multiplicative functions, denoted by $M$, with this operation is an abelian group.

We shall call an arithmetic function $f$ an $n$-ic function if $f$ is the convolution of $n$ linear functions. We shall also call an arithmetic function $h$ a rational function of order $(n, m)$ if $h$ is the convolution of an $n$-ic function $f$ and the inverse of an $m$-ic function $g$, where $g^{-1} \circ g(k)=\alpha(k) \equiv[1 / k]$. The term rational function of order $(r, s)$ seems to originate with Vaidyanathaswamy (1931), who examined this type of multiplicative functions. Note that a rational function of order $(1,1)$ is commonly called a totient. We shall use the convention that $\alpha$ is a 0 -ic function. Hence, an $n$-ic function $f=f \circ \alpha$ can also be considered as a rational function of order $(n, 0)$ and the inverse of an $m$-ic function can be said to be a rational function of order $(0, m)$. If se let

$$
R=\left\{f \mid f \text { is a rational function of order }(n, m), n, m \in Z^{+} \cup\{0\}\right\},
$$

then it is an easy matter to show $(R, \circ)$ is a subgroup of $(M, 0)$.

It is the purpose of this paper to exhibit characterizations of $n$-ic functions and rational functions of order $(1, n)$ as well as examine rational functions of an arbitrary order $(n, m)$. 


\section{2. $n$-ic Functions}

In this section we will prove a characterization of $n$-ic functions that will be exceedingly useful in the remaining sections. An example of an $n$-ic fnuction that has been studied extensively is $\mathfrak{t}_{0}^{(n)}(m)=\mathfrak{t}_{0} \circ \mathfrak{l}_{0} \circ \cdots \circ \mathfrak{l}_{0}(m)$ ( $n$ times), where $\mathfrak{l}_{0}(m)=1$ for all $m \in Z^{+}$. This function is seen to be the number of ways $m$ can be expressed as a product of $n$ factors (including unity factors) (see Dickson (1919; page 308)), Beumer (1962) and Potter (1968)). The following lemma, which will be used to prove Theorem 2.2 , is easily proved by a straightforward argument.

LEMMA 2.1. Let $f$ be an arithmetic function. Then $f$ is $n$-ic if and only if $f$ is multiplicative and for every prime $p$ and all $a \in Z^{+}$.

$$
f^{-1}\left(p^{a}\right)=-f_{1}(p) g^{-1}\left(p^{a-1}\right)+g^{-1}\left(p^{a}\right)
$$

where $f_{1}$ is a linear function, $g$ is a $(n-1)$-ic function, and $f^{-1}$ is the inverse of $f$ with respect to "०".

Proof. If $f$ is $n$-ic, then there exists functions $f_{1}$ and $g$ such that $f_{1}$ is linear, $g$ is $(n-1)-i c$, and $f^{-1}=f_{1}^{-1} \circ g^{-1}$. Therefore, since $f_{1}$ is linear

$$
\begin{aligned}
f^{-1}\left(p^{a}\right) & =f_{1}^{-1}(p) g^{-1}\left(p^{a-1}\right)+g^{-1}\left(p^{a}\right) \\
& =-f_{1}(p) g^{-1}\left(p^{a-1}\right)+g^{-1}\left(p^{a}\right)
\end{aligned}
$$

for $p$ a prime and $a$ any natural number.

Conversely, to show $f$ is $\boldsymbol{n}-\boldsymbol{i c}$, since $f$ is multiplicative, we need only show that $f^{-1}\left(p^{a}\right)=f_{1}^{-1} \circ g^{-1}\left(p^{a}\right)$ for an arbitrary prime $p$ and $a \in Z^{+}$. For $p$ a prime and $a \in Z^{+}$, since $f_{1}$ is linear, $f_{1}^{-1}\left(p^{a}\right)=0$ for all $a \geqq 2$ (Apostol (1971)). Thus,

$$
\begin{aligned}
f^{-1}\left(p^{a}\right) & =-f_{1}(p) g^{-1}\left(p^{a-1}\right)+g^{-1}\left(p^{a}\right) \\
& =g^{-1}\left(p^{a}\right)+f_{1}^{-1}(p) g^{-1}\left(p^{a-1}\right)+f_{1}^{-1}\left(p^{2}\right) g^{-1}\left(p^{a-2}\right)+\cdots+f_{1}^{-1}\left(p^{a}\right) \\
& =g^{-1} \circ f_{1}^{-1}\left(p^{a}\right) .
\end{aligned}
$$

The necessity of the following theorem was first proven by Vaidyanathaswamy (1930). It is beliewed that the proof of the sufficiency appears here for the first time. Note that this theorem is an analogous generalization of the well-known characterization for linear functions $(n=1)$.

THEOREM 2.2. Let $f$ be an arithmetic function. A necessary and sufficient condition for $f$ to be $n-i c$ is that $f$ is multiplicative and, for each prime $p$, $f^{-1}\left(p^{a}\right)=0$ for all $a>n$.

Proof. The reader is referred to Vaidyanathaswamy (1930) for the proof that the condition is necessary. The sufficiency part of the proof will be proven by 
induction on $n$. This is known to be true for $n=1$ (see Apostol (1971)). Suppose $n>1$ and the theorem is true for $k \leqq n-1$. We will construct a linear function $f_{1}$ and an $(n-1)$-ic function $g$ such that for every prime $p$ and every $a \in Z^{+}$, we have

$$
f^{-1}\left(p^{a}\right)=-f_{1}(p) g^{-1}\left(p^{a-1}\right)+g^{-1}\left(p^{a}\right) .
$$

For a fixed prime $p$, the construction procedes as follows. Let $A=A(p)$ be the least integer such that $f^{-1}\left(p^{a}\right)=0$ for all $a>A$. Clearly $n \geqq A \geqq 0$ and $f^{-1}\left(p^{A}\right) \neq 0$. If $A=0$, then the matter is trivial - take $g(1)=f_{1}(1)=1$ and $g\left(p^{a}\right)=f_{1}\left(p^{a}\right)=0$ for all $a>0$. If $A>0$, consider the polynomial equation (in $x$ )

$$
0=\sum_{k=0}^{A-1}(-1)^{k} x^{A-k} f^{-1}\left(p^{k}\right)-(-1)^{A} f^{-1}\left(p^{A}\right) .
$$

This equation of degree $A$ with non-zero constant term has a nonzero solution say $x=B$. Define $g$ (a multiplicative function) inductively at the powers of this prime $p$ by the first order difference equation

$$
g^{-1}\left(p^{a}\right)+B g^{-1}\left(p^{a-1}\right)=f^{-1}\left(p^{a}\right), \quad a=1,2,3, \cdots
$$

with the initial condition $g^{-1}\left(p^{A-1}\right)=f^{-1}\left(p^{A}\right) / B$. The solution of this difference equation is $g^{-1}\left(p^{a-1}\right)=0$ for all $a>A-1$, and

$$
\begin{array}{r}
g^{-1}\left(p^{A-j}\right)=\sum_{k=1}^{j-1}(-1)^{k+1} B^{-k} f^{-1}\left(p^{A-j+k}\right)+(-1)^{j+1} B^{-j} f^{-1}\left(p^{A}\right), \\
j=2,3, \cdots .
\end{array}
$$

(Notice that the solution for $j=A$ is valid since $B$ is a solution of (2.1)). Now define the linear function $f_{1}$ at this prime $p$ to be $f_{1}(p)=-B$.

When the construction is completed at each prime, we have $f_{1}$ linear and $g^{-1}\left(p^{a}\right)=0$ for all $a \geqq A=A(p)$. Therefore by the induction assumption, since $A(p) \leqq n$ for all primes $p, g$ is $(n-1)-i c$, and (2.2) clearly shows that the condition of Lemma 2.1 is satisfied. Thus $f$ is $n-i c$.

\section{Busche-Ramanujan identities of order $N$}

A multiplicative function $f$ is said to admit a Busche-Ramanujan identity if there exists a multiplicative function $F$ such that for all positive integers $m$ and $n$,

$$
f(m n)=\sum_{d \mid(m, n)} f(m / d) f(n / d) F(d) .
$$

This definition is due to Vaidyanathaswamy (1931), who showed that the only multiplicative functions which admit Busche-Ramanujan identities are quadratic functions (in our notation $2-i c$ functions). Busche-Ramanujan identities have 
been also studied by Ramanathan (1943) and McCarthy (1960). This section extends their results to $N-i c$ functions.

Definition 3.1. A multiplicative function $f$ is said to admit a BuscheRamanujan identity of order $N$, if there exists a multiplicative function $F$ such that

$$
f(m n)=\sum_{d \mid(m, C m)} \sum_{e \mid(n, C m / d)} f(m / d) f(n / e) F(d e)
$$

where $C_{m}=p_{1} p_{2} \cdots p_{r}$ if $m=p_{1}^{a_{1}} p_{2}^{a_{2}} \cdots p_{r}^{a_{r}}, a_{i}>0$ for $1 \leqq i \leqq r, p_{1}, p_{2}, \cdots, p_{r}$ distinct primes.

We now state a theorem that is due to Vaidyanathaswamy (1931; page 645). For a proof that depends only the fact that $f \circ f^{-1}\left(p^{a}\right)=\alpha\left(p^{a}\right)$ see Gioia (1962).

THEOREM 3.2. Suppose that $f$ is a multiplicative function. For a fixed prime $p, a \in Z^{+}$, and $b \in Z^{+}$,

$$
f\left(p^{a+b}\right)=(-1) \sum_{i=0}^{a} \sum_{j=0}^{b} f\left(p^{a-i}\right) f\left(p^{b-j}\right) f^{-1}\left(p^{i+j}\right) .
$$

THeOREM 3.3. A multiplicative function $f$ is $n-i c$ if and only if for each prime $p$, all $a \in Z^{+}$, and all $b \in Z^{+}$,

$$
f\left(p^{a+b}\right)=(-1) \sum_{d \mid\left(p^{a}, p^{n}\right)} \sum_{e \mid\left(p^{b}, p^{n / d}\right)} f\left(p^{a} / d\right) f\left(p^{b} / e\right) f^{-1}(d e) .
$$

Proof. If $f$ is $n-i c$, then (3.3) follows directly from Theorems 2.2 and 3.2.

Conversely, it suffices to show that $f^{-1}\left(p^{n+k}\right)=0$ for $k \geqq 1$. Letting $a=n$ and $b=1$, we obtain the following in (3.3):

$$
\begin{aligned}
f\left(p^{n+1}\right) & =(-1) \sum_{d \mid\left(p^{n}, p^{n}\right)} \sum_{e \mid\left(p, p^{n / d}\right)} f\left(p^{n} / d\right) f(p / e) f^{-1}(d e) \\
& =(-1) \sum_{i=0}^{n-1} f\left(p^{n-i}\right)\left\{f(p) f^{-1}\left(p^{i}\right)+f^{-1}\left(p^{i+1}\right)\right\}+(-1) f(p) f^{-1}\left(p^{n}\right) \\
& =(-1) f(p) \sum_{i=0}^{n} f\left(p^{n-i}\right) f^{-1}\left(p^{i}\right)+(-1) \sum_{i=0}^{n-1} f\left(p^{n-i}\right) f^{-1}\left(p^{i+1}\right) \\
& =(-1) \sum_{j=1}^{n} f\left(p^{n+1-j}\right) f^{-1}\left(p^{j}\right) .
\end{aligned}
$$

Thus,

$$
\sum_{j=0}^{n} f\left(p^{n+1-j}\right) f^{-1}\left(p^{j}\right)=0=\sum_{j=0}^{n+1} f\left(p^{n+1-j}\right) f^{-1}\left(p^{j}\right) .
$$

Therefore, $f^{-1}\left(p^{n+1}\right)=0$. If we assume that $f^{-1}\left(p^{n+x}\right)=0$ for $1 \leqq x \leqq k$ and 
we let $a=n+(k-1)$ and $b=1$ in (3.3), then, using the same rearrangements as the case $k=1$, we obtain

$$
0=\sum_{j=0}^{n} f\left(p^{n+k-j}\right) f^{-1}\left(p^{j}\right)=\sum_{j=0}^{n} f\left(p^{n+k-j}\right) f^{-1}\left(p^{j}\right)+f^{-1}\left(p^{n+k}\right) .
$$

Therefore, the theorem is proven by induction on $k$ for each prime $p$. It is known that, for $m$ and $n$ positive integers,

$$
C(m, n)= \begin{cases}(-1)^{k} & \text { if } m \text { and } n \text { have the same } \\ & k \text { distinct prime divisors, } \\ 0 & \text { otherwise }\end{cases}
$$

is multiplicative in both variables (Vaidyanathaswamy (1931; page 645)). W enow prove the following lemma.

LEMMA 3.4. If $f$ is multiplicative and a function $g(m, n)$ is defined by

$$
g(m, n)=\sum_{d \mid(m, C m)} \sum_{e !(n, C m / d)} f(m / d) f(n / e) f^{-1}(d e) C(d, e),
$$

then $g(m, n)$ is multiplicative in both variables.

Proof. Let $(a c, b d)=1$. We wish to show that $g(a, b) g(c, d)=g(a c, b d)$. $g(a, b) g(c, d)$

$$
\begin{aligned}
= & \sum_{w \mid(a, C a)} \sum_{x \mid(b, C a / w)} f(a / w) f(b / x) f^{-1}(w x) C(w, x) \times \\
& \sum_{y \mid(c, C c)} \sum_{z \mid(d, C c / y)} f(c / y) f(d / z) f^{-1}(y z) C(y, z) \\
= & \sum_{w \mid(a, C a)} \sum_{x \mid(b, C a / w)} f(a / w) f(c / y) f(b / x) f(d / z) f^{-1}(w x) f^{-1}(y z) C(w, x) C(y, z) \\
y \mid(c, C c) & z \mid(a, C c / y) \\
= & \sum_{w y \mid(a c, C a c)}^{N} \quad \sum_{x z \mid(b d, C a c / w y)} \\
= & g(a c, b d) .
\end{aligned}
$$

THEOREM 3.5. An arithmetic function $f$ is $N-i c$ if and only if $f$ is multiplicative and

$$
f(m n)=\sum_{d \mid(m, C n)} \sum_{e \mid\left(n, C_{m} / d\right)} f(m / d) f(n / e) f^{-1}(d e) C(d, e)
$$

for all $m$ and $n$ in $Z^{+}$

Proof. By Lemma 3.4 it suffices to consider the case where the argument is a power of a prime. Thus, let $m=p^{a}$ and $n=p^{b}$ where $p$ is a prime and $a$ and $b$ 
are non-negative integers. If both $a$ and $b$ are in $Z^{+}$, then we are finished by Theorem 3.3. If $a=0$ or $b=0$, then $C(d, e)=0$ unless $d=e=1$. Hence, the result holds for all non-negative $a$ and $b$.

\section{Quadratic functions}

Quadratic functions have been studied more than any other type of $n-i c$ functions (with the possible exception of linear functions) chiefly because $\sigma_{s}(n)=\mathfrak{l}_{s} \circ \mathfrak{l}_{0}(n)$ are quadratic functions. In addition to the characterizations of quadratic functions that we have given in sections two and three, we state the following characterization (see McCarthy (1960)).

Theorem 4.1. A multiplicative function $f$ is quadratic if and only if, for all primes $p$ and all $a \in Z^{+}$,

$$
f\left(p^{a+1}\right)=f\left(p^{a}\right) f(p)-f\left(p^{a-1}\right) f^{-1}\left(p^{2}\right) .
$$

In this section we give yet another characterization. In general, given an $n-i c$ function $f$, it is easy to see that for each prime $p, f$ is completely determined by $f^{-1}(p), f^{-1}\left(p^{2}\right), \cdots, f^{-1}\left(p^{n}\right)$. That is, there exists a function $F$ such that

$$
f\left(p^{m}\right)=F\left(f^{-1}(p), f^{-1}\left(p^{2}\right), \cdots, f^{-1}\left(p^{n}\right), p^{m}\right)
$$

for all $m \in Z^{+}$. However, for an arbitrary $n$ this function $F$ is quite complicated. For the case $f$ quadratic we explicitly give this function and also show that if $f$ is multiplicative and $f$ satisfies (4.1) for the given $F$, then $f$ is quadratic.

THEOREM 4.2. Assume $f$ is a multiplicative function. Then $f$ is a quadratic function if and only if for every prime $p$ and every $n \in Z^{+}$

$$
f\left(p^{n}\right)=\sum_{0 \leqq i \leqq[n / 2]}(-1)^{n+i}\left(\begin{array}{c}
n-i \\
i
\end{array}\right)\left(f^{-1}(p)\right)^{n-2 i}\left(f^{-1}\left(p^{2}\right)\right)^{i} .
$$

Before we prove this theorem we prove the following lemma.

LEMMA 4.3. Assume $f$ is an arithmetic function possessing an inverse. Then

$$
F\left(p^{n}\right)=-f^{-1}(p) F\left(p^{n-1}\right)-f^{-1}\left(p^{2}\right) F\left(p^{n-2}\right)
$$

for $n \geqq 2$, where $F\left(p^{n}\right)$ equals the summation in (4.2).

Proof. This lemma will be proven in two cases.

Case (1) $-n$ is even. Let $n=2 l+2$. Then

$$
\begin{aligned}
& -f^{-1}(p) F\left(p^{2 l+1}\right)-f^{-1}\left(p^{2}\right) F\left(p^{2 l}\right) \\
& \quad=\sum_{i=0}^{l}(-1)^{2 l+2+i}\left(\begin{array}{c}
2 l+1-i \\
i
\end{array}\right)\left(f^{-1}(p)\right)^{2 l+2-2 i}\left(f^{-1}\left(p^{2}\right)\right)^{i}
\end{aligned}
$$




$$
\begin{aligned}
& +\sum_{i=0}^{l}(-1)^{2 l+1+i}\left(\begin{array}{c}
2 l-i \\
i
\end{array}\right)\left(f^{-1}(p)\right)^{2 l-2 i}\left(f^{-1}\left(p^{2}\right)\right)^{i+1} \\
= & \left(\begin{array}{c}
2 l+1 \\
0
\end{array}\right)(-1)^{2 l+2}\left(f^{-1}(p)\right)^{2 l+2} \\
& +\sum_{i=1}^{l}(-1)^{2 l+2+i}\left(\left(\begin{array}{c}
2 l+1-i \\
i
\end{array}\right)+\left(\begin{array}{c}
2 l+1-i \\
i
\end{array}\right)\right)\left(f^{-1}(p)\right)^{2 l+2-2 i} \times \\
& \left(f^{-1}\left(p^{2}\right)\right)^{i}+(-1)^{2 l+2+(l+1)}\left(\begin{array}{c}
2 l-l \\
l
\end{array}\right)\left(f^{-1}(p)\right)^{2 l-2 l}\left(f^{-1}\left(p^{2}\right)\right)^{l+1} \\
= & \sum_{i=0}^{l+1}(-1)^{2 l+2+i}\left(\begin{array}{c}
2 l+2-i \\
i
\end{array}\right)\left(f^{-1}(p)\right)^{2 l+2-2 i}\left(f^{-1}\left(p^{2}\right)\right)^{i} \\
= & F\left(p^{2 l+2}\right) .
\end{aligned}
$$

Case (2) $-n$ is odd. Let $n=2 l+1$.

$$
\begin{aligned}
- & f^{-1}(p) F\left(p^{2 l}\right)-f^{-1}\left(p^{2}\right) F\left(p^{2 l-1}\right) \\
= & \sum_{i=0}^{l}(-1)^{2 l+1+i}\left(\begin{array}{c}
2 l-i \\
i
\end{array}\right)\left(f^{-1}(p)\right)^{2 l+1-2 i}\left(f^{-1}\left(p^{2}\right)\right)^{i} \\
& +\sum_{i=0}^{l-1}(-1)^{2 l+i}\left(\begin{array}{c}
2 l-1-i \\
i
\end{array}\right)\left(f^{-1}(p)\right)^{2 l-1-2 i}\left(f^{-1}\left(p^{2}\right)\right)^{i+1} \\
= & (-1)^{2 l+1}\left(\begin{array}{c}
2 l \\
0
\end{array}\right)\left(f^{-1}(p)\right)^{2 l+1} \\
& +\sum_{i=1}^{l}(-1)^{2 l+1+i}\left(\left(\begin{array}{c}
2 l-i \\
i
\end{array}\right)+\left(\begin{array}{c}
2 l-i \\
i-1
\end{array}\right)\right)\left(f^{-1}(p)\right)^{2 l+1-2 i}\left(f^{-1}\left(p^{2}\right)\right)^{i} \\
= & \sum_{i=0}^{l}(-1)^{2 l+1+i}\left(\begin{array}{c}
2 l+1-i \\
i
\end{array}\right)\left(f^{-1}(p)\right)^{2 l+1-2 i}\left(f^{-1}\left(p^{2}\right)\right)^{i} \\
= & F\left(p^{2 l+1}\right) .
\end{aligned}
$$

Proof of Theorem 4.2. Assume $f$ is quadratic. Equation (4.2) is easily seen to hold for $n=1$ for every prime $p$. Let $p$ be an arbitrary prime and assume Equation (4.2) holds for all powers of $p$ less than $n$. By Theorem 4.1

$$
f(p)^{n}=-f^{-1}(p) f\left(p^{n-1}\right)-f^{-1}\left(p^{2}\right) f\left(p^{n-2}\right)
$$

for all $n \geqq 2$. By the induction assumption and Lemma 4.2

$$
\begin{aligned}
& f\left(p^{n}\right)=-f^{-1}(p) f\left(p^{n-1}\right)-f^{-1}\left(p^{2}\right) f\left(p^{n-2}\right) \\
& =-f^{-1}(p) F\left(p^{n}\right)-f^{-1}\left(p^{2}\right) F\left(p^{n-2}\right) \\
& =F\left(p^{n}\right) .
\end{aligned}
$$


Conversely, assume $f$ satisfies Equation (4.2) for all $n$. By Lemma 4.2 for all $n \geqq 2$

$$
f\left(p^{n}\right)=-f^{-1}(p) f\left(p^{n-1}\right)-f^{-1}\left(p^{2}\right) f\left(p^{n-2}\right) .
$$

It is now an easy inductive argument that shows $f^{-1}\left(p^{a}\right)=0$ for all $a>2$.

We obtain some interesting identities if we use specific quadratic functions in Equation (4.2). Two well-known quadratic functions are the tau function (the number of divisors of a natural number) and sigma function (the sum of the divisors of a natural number). For the tau function

$$
\tau\left(p^{n}\right)=n+1=\sum_{0 \leqq i \leqq[n / 2]}(-1)^{n+i}\left(\begin{array}{c}
n-i \\
i
\end{array}\right) 2^{n-2 i},
$$

and for the sigma function

$$
\sigma\left(p^{n}\right)=\sum_{0 \leqq i \leqq[n / 2]}(-1)^{n+i}\left(\begin{array}{c}
n-i \\
i
\end{array}\right)(1+p)^{n-2 i} p^{i}
$$

\section{Rational functions}

An arithmetic function $f$ is called a totient if $f$ is the convolution of a linear function and the inverse of a linear function. Recently Wall and Hsu (1972) showed that a multiplicative function $f$ is a totient if and only if for each prime $p, f(p), f\left(p^{2}\right), \cdots$ is a geometric progression. We define an arithmetic function $f$ to be a rational function of order $(1, n)$ if $f$ is the convolution of a linear function and the inverse of an $n-i c$ function. We obtain the following result which is analogous to Wall and Hsu's theorem.

THEOREM 5.1. A multiplicative function $F$ is a rational function of order $(1, n)$ if and only iffor each prime $p, F\left(p^{n}\right), F\left(p^{n+1}\right), \cdots$ is a geometric progression.

Proof. If $F$ is a rational function of order $(1, n)$, then there exists a linear function $f$ and an $n-i c$ function $g$ such that $F=f \circ g^{-1}$. Let

$$
F\left(p^{n}\right)=f \circ g^{-1}\left(p^{n}\right)=a .
$$

Since $f$ is linear and $g$ is $n-i c$, for all $k \in Z^{+}$

$$
\begin{aligned}
F\left(p^{n+k}\right) & =\sum_{i=0}^{n+k} f\left(p^{n+k-i}\right) g^{-1}\left(p^{i}\right) \\
& =(f(p))^{k} \sum_{i=0}^{n} f\left(p^{n-i}\right) g^{-1}\left(p^{i}\right) \\
& =(f(p))^{k} \sum_{i=0}^{n} f\left(p^{n-i}\right) g^{-1}\left(p^{i}\right) \\
& =(f(p))^{k} a .
\end{aligned}
$$


Conversely, let $F\left(p^{n+k}\right)=r^{k} a, k \geqq 0$. Let $f$ be a linear function such that $f(p)=r$. Consider the following system of $n$ equations in the $n$ unknowns, $x_{1}, x_{2}, \cdots, x_{n}$ :

$$
\begin{aligned}
r^{n-1} x_{1}+r^{n-2} x_{2}+\cdots+r x_{n-1}+x_{n} & =F\left(p^{n}\right)-r^{n} \\
r^{n-2} x_{1}+r^{n-3} x_{2}+\cdots+x_{n-1} & =F\left(p^{n-1}\right)-r^{n-1} \\
\cdot . . . & \\
r x_{1}+x_{2} & =F\left(p^{2}\right)-r^{2} \\
x_{1} & =F(p)-r .
\end{aligned}
$$

Since

$$
\operatorname{det}\left[\begin{array}{lllll}
r^{n-1} & r^{n-2} & \cdots & r & 1 \\
r^{n-2} & r^{n-3} & \cdots & 1 & \\
& \cdot & \cdot & & \\
r & 1 & & & \\
1 & & &
\end{array}\right]=(-1)^{(n-1) n / 2} \neq 0,
$$

there exists a unique solution of this system of equations, say $\left(y_{1}, y_{2}, \cdots, y_{n}\right)$. Let $g$ be a multiplicative function such that

$$
g^{-1}(p)=y_{i}, 1 \leqq i \leqq n \text {, and } g^{-1}\left(p^{n+k}\right)=0
$$

for $k \in Z^{+}$. By Theorem $2.2, g$ is $n-i c$. For $1 \leqq j \leqq n$, one sees that

$$
F\left(p^{j}\right)=f \circ g^{-1}\left(p^{j}\right)
$$

and for $n+k=j$

$$
\begin{aligned}
F\left(p^{j}\right) & =F\left(p^{n+k}\right)=r^{k} a=r^{k} F\left(p^{n}\right) \\
& =(f(p))^{k} f \circ g^{-1}\left(p^{n}\right) \\
& =f \circ g^{-1}\left(p^{n+k}\right) .
\end{aligned}
$$

Therefore, $F$ is a rational function of order $(1, n)$.

\section{The subgroup of rational functions}

We shall call a function that is the convolution of an $n-i c$ function and the inverse of an $m-i c$ function a rational function of order $(n, m)$. If we use the convention that $\alpha(\alpha(n)=[1 / n])$ is an $0-i c$ function, then an $n-i c$ function is also a rational function of order $(n, 0)$ and the inverse of an $m-i c$ function is a rational function of order $(0, m)$. One notices that $\alpha$ is the only rational function 
of order $(0,0)$. It is easy to see that if we let $R=\{f \mid f$ is a rational function of order $(n, m), n \geqq 0, m \geqq 0\}$, then $(T, \circ)$ is a subgroup of $(M, \circ)$.

As a consequence of Theorem 2.2 we prove that rational functions of order $(n, m)$ satisfy the following theorem.

THEOREM 6.1. If $F$ is a rational function of order $(n, m)$, then for each prime $p$ and each $k \geqq \max \{n, m\}=N$ there exists an $n-i c$ function $f$ and an $m-i c$ function $g$ such that

$$
F\left(p^{k}\right)=\sum_{i=1}^{N} f\left(p^{k-i}\right)\left(g^{-1}\left(p^{i}\right)-f^{-1}\left(p^{i}\right)\right) .
$$

Proof. Note that if we use the convention that $f\left(p^{x-y}\right)=0$ for $x<y$, then the restriction that $k \geqq N$ can be dropped.

Since $F$ is a rational function of order $(n, m)$, there exist functions $f$ and $g$ such that $f$ is $n-i c, g$ is $m-i c$, and $F=f \circ g^{-1}$. Hence

$$
\begin{aligned}
F\left(p^{k}\right) & =\sum_{i=0}^{k} f\left(p^{k-i}\right) g^{-1}\left(p^{i}\right) \\
& =\sum_{i=1}^{k} f\left(p^{k-i}\right) g^{-1}\left(p^{i}\right)-\sum_{i=1}^{k} f\left(p^{k-i}\right) f^{-1}\left(p^{i}\right) \\
& =\sum_{i=0}^{k} f\left(p^{k-i}\right)\left(g^{-1}\left(p^{i}\right)-f^{-1}\left(p^{i}\right)\right) .
\end{aligned}
$$

Since $k \geqq N, g^{-1}\left(p^{i}\right)=f^{-1}\left(p^{i}\right)=0$ for $N<i \leqq k$. Therefore, the theorem follows.

\section{Remark}

If we replace the Dirichlet convolution by the basic sequence convolution (Goldsmith (1969)), then, using the severance class theory developed by the authors, Carroll and Gioia (1971), analogous results for all the theorems in this paper hold.

\section{References}

T. M. Apostol (1971), 'Some properties of completely multiplicative arithmetic functions', Amer. Math. Monthly 78, 266-271.

M. Beumer (1962), 'The arithmetical function $\tau_{k}(n)$ ', Amer. Math. Monthly 69, 777-781.

T. B. Carroll and A. A. Gioia (1971), 'On extended linear functions', Notices Amer. Math. Soc. 18bf, Abstract \#71T-A161, 799.

L. E. Dickson (1919), History of the Theory of Numbers, Volume I (Washington, Carnegie Institution, 1919).

A. A. Gioia (1962). 'On an identity for multiplicative functions', Amer. Math. Monthly 79, 988-991.

D. L. Goldsmith (1969), 'A generalization of some identities of Ramanujan', Rend. Mat. (Serie vi) 2, 473-479. 
P. J. McCarthy (1960), 'Busche-Ramanujan identities', Amer. Math. Monthly 67, 966-970.

M. Potter (1968), Extensions of the Sigma and Tau Functions, (Unpublished Specialist's project, Western Michigan University, Kalamazoo, Michigan, 1968).

K. G. Ramanathan (1943), 'Multiplicative arithmetic functions', Indian Math. Soc. 7, 111-116.

R. Vaidyanathaswamy (1930), 'The identical equations of the multiplicative function', Bull. Amer. Math. Soc. 36, 762-772.

R. Vaidyanathaswamy (1931), 'The theory of multiplicative arithmetical functions', Trans. Amer. Math. Soc. 33, 579-662.

C. R. Wall and T. S. Hsu (1972), 'A characterization of totients', Notices Amer. Math. Soc. 19, Abstract \# 72T-A175, 567.

\section{Western Michigan University}

Kalamazoo

Michigan 49001

U.S.A. 